\title{
The Influence of Acetabular Morphologic Variations on the Cup Position in Total Hip Arthroplasty for Crowe type III Dysplastic Hip
}

Junmin Shen

Chinese PLA General Hospital

\section{Ti Zhang}

Chinese PLA General Hospital

\section{Yu Dong}

Chinese PLA General Hospital

\section{Yanchao Zhang}

Chinese PLA General Hospital

\section{Yonggang Zhou}

Chinese PLA General Hospital https://orcid.org/0000-0002-7304-4691

Lingfei Guo ( $\nabla$ guolingfei316@126.com )

Chinese PLA General Hospital https://orcid.org/0000-0003-3028-4545

\section{Research article}

Keywords: Morphologic variations, Crowe III, Total hip arthroplasty, Cup position

Posted Date: November 1st, 2021

DOI: https://doi.org/10.21203/rs.3.rs-1030386/v1

License: (9) (i) This work is licensed under a Creative Commons Attribution 4.0 International License. Read Full License 


\section{Abstract}

Background: We aimed to (1) evaluate the acetabular morphologic variations of Crowe III hips; (2) study the influence of different morphologies on the cup position in total hip arthroplasty.

Methods: From November 2008 to February 2019, we retrospectively evaluated 101 patients (110 hips) with Crowe III developmental dysplasia of the hip. We classified Crowe III hips into two subtypes, the IIIA when the acetabular roof was extensively deficient and the junction between the false and the true acetabulum was indistinct, and the IIIB when there is a significant crest between the false and the true acetabulum. Based on the radiographs, we measured the morphological characteristic of the acetabulum and the postoperative cup position.

Results: The false acetabulum of IIIB hips had larger Tonnis angle and smaller center-edge angle than the IIIA hips. The width of true acetabular roof in the IIIB hips was thicker than the IIIA group. Fifty-one (100\%) IIIA hips and 48 (81.4\%) IIIB hips were reconstructed using high hip center while 11 (18.6\%) IIIB hips were reconstructed anatomically. The mean vertical distance of center of rotation in the IIIA group was $33.5 \pm 4.5 \mathrm{~mm}$ while it was $31.2 \pm 6.3 \mathrm{~mm}$ in the IIIB group $(p=0.040)$. The vertical distance of the hip center was positively correlated with the height of dislocation in the IIIA group $(r=0.493, p<0.001)$. According to the four-zone system, in the IIIA group, 5 hips were located in the inferomedial zone, 23 hips in the superomedial zone, 22 hips in the superolateral zone and 1 hip in the inferolateral zone. In the IIIB group, the corresponding numbers were respectively $15,17,1$ and 5 . Compared with the IIIA group, there were more IIIB hips located in the inferomedial zone $(p=0.008)$ and less in the superolateral zone $(p=0.033)$.

Conclusions: There are distinct morphologic subtypes based on the relationship between the false and the true acetabulum. More bone stock located in the superior wall of the true acetabulum can bring more possibilities for anatomical reconstruction, and lower the height of center of rotation when using high hip center.

\section{Background}

Developmental dysplasia of the hip (DDH) is a condition which encompasses a wide spectrum of morphological abnormalities of the acetabulum and femur[1]. According to the amount of cephalic migration of the femoral head, Crowe et al.[2] proposed a four-type classification which is currently the most prevalent classification system used in clinical practice and various studies. However, the pathoanatomy of acetabulum is variable, and the classification of Crowe cannot distinguish the difference between acetabular morphologies.

Crowe type III DDH, defined as proximal dislocation of $15-20 \%$ of pelvic height, particularly brings technical challenge to surgeons in total hip arthroplasty (THA). We have observed distinct morphologic variations of the deformed acetabulum in the Crowe III hips based on the anteroposterior (AP) pelvis radiographs. When the bone deficiency of the true acetabular roof was limited, a significant crest between the false and the true acetabulum can be observed, and the false acetabulum can present with the 
characteristic of shallow depth and relatively large inclination (Fig. 1A-B). When the true acetabular roof was extensively deficient, the junction between the false and the true acetabulum was indistinct (Fig. 1CD). Additionally, the native host bone above the true acetabulum was unable to provide adequate boneimplant contact to achieve anatomical reconstruction, unless the structural bone grafts or high hip center $(\mathrm{HHC})$ technique were utilized[3, 4, 5].

Previously, some studies have carried out the acetabular morphology evaluation and measurement of Crowe III hips. Yang et al.[6] reported that the Crowe III hips tended to have a superior segmental deficiency of the acetabulum as a result of the overlap of the true and false acetabulum. Through computer simulation, Zheng et al.[7] found the dysplastic acetabulum in Crowe III hips had larger superoinferior and anteroposterior diameters, and the median vertical height of the cup center was $24 \mathrm{~mm}$ to achieve $80 \%$ of the coverage. Whereas, there was no literature that paid attention to the acetabular morphologic variations of Crowe III hips, of which the influence on the cup position was unknown as well.

Therefore, the present study aimed to (1) evaluate the acetabular morphologic variations of Crowe III hips by measurement; (2) study the influence of different morphologies on the cup position in THA.

\section{Materials And Methods}

Institutional review board approval was obtained before initiation of this retrospective case-series study. During the periods from November 2008 to February 2019, a total of 141 patients who were diagnosed with Crowe type III DDH and treated with cementless THA were identified from our departmental database. We excluded those patients who have the history of previous acetabular hip surgery (38 hips in 36 patients) and neuromuscular disease (4 hips in 4 patients). These exclusions left us with 110 hips in 101 patients. In this study, there were 85 women (93 hips) and 16 men (17 hips) with a mean age of 45.5 \pm 12.2 years (range, $21-71$ years). Fifty-one right hips and 59 left hips were surgically treated. The mean body mass index (BMI) was $23.8 \pm 3.4 \mathrm{~kg} / \mathrm{m}^{2}$ (range, $15.2-32.9 \mathrm{~kg} / \mathrm{m}^{2}$ ). The mean follow-up time was 5.7 \pm 2.9 years (range, $1.4-11.7$ years).

Based on the preoperative AP radiographs, we classified Crowe III hips into 2 subtypes (Table 1). When the roof of the true acetabulum was extensively deficient and the junction between the false and the true acetabulum was indistinct, the hip was identified as IIIA. When there was a significant crest between the false and the true acetabulum, the hip was identified as IIIB. The radiographs were examined by two experienced orthopedic surgeons independently, and this procedure was repeated by the same surgeon after 2 months. Assessment of intra- and interobserver consistency was accomplished using the kappa coefficient. The interobserver reliability between two observers was 0.837 , and the intraobserver reliability between the two evaluations of the same surgeon was 0.909 .

\section{Operative Technique}


All operations were conducted by one senior orthopedic surgeon, through a modified Kocher-Langenbeck posterolateral approach, with the patient in the lateral decubitus position. During the preparation of the acetabulum, the anatomical reconstruction was the primary aim. However, in most cases, bone deficiencies made it difficult for the cup to acquire stable initial fixation in the anatomic position. To address this problem, the HHC technique which is conducive to achieve sufficient cup coverage was utilized. Structural bone graft was not considered by the reason of complexity and time-consuming.

We used three types of acetabular component. A Pinnacle cup (DePuy, Warsaw, IN, USA) was used in 77 hips, a Betacup (Link, Hamburg, Germany) in 21 hips, and a Duraloc (DePuy) cup in 12 hips. We used mainly two cementless stem, Corail (DePuy) and S-ROM (DePuy). Corail stem was used in 54 hips while S-ROM stem was used in 44 hips. We also used 5 Accolade (Stryker, Mahwah, NJ, USA) stems, 6 LCU (Link) stems and 1 Ribbed (Link) stem in another 12 hips. The mean outer diameter of the acetabular cup was $49.3 \mathrm{~mm}$ (range, 44-58mm). We used a $28-\mathrm{mm}$ head in 29 hips, a $32-\mathrm{mm}$ head in 48 hips, and a 36$\mathrm{mm}$ head in 43 hips. The articulating surfaces used were ceramic-on-ceramic in all hips.

\section{Radiographic evaluation}

Conventional radiographs were achieved for every patient in preoperative, postoperative immediately and last follow-up assessment. The height of dislocation was defined as the vertical distance from the medial head-neck junction to the inter-teardrop line. The horizontal distance from the most superior point of the true acetabulum to the line perpendicular to the inter-teardrop line was measured, and the width of true acetabular roof was calculated as the ratio between this distance and $1 / 5$ of the height of pelvic (represent the height of femoral head). The morphological characteristic of the false acetabulum was measured by the acetabular index angle (Tonnis angle), which was defined as the angel between horizontal line at level of medial edge of the weightbearing surface of the false acetabulum and line tangential to medial and lateral edges of the weightbearing surface[8]. The center-edge (CE) angle, defined as the angle between vertical line through femoral head center and line tangential to lateral margin of false acetabulum, was measured to show the coverage of the femoral head by the false acetabulum[9] (Fig.2).

The cup position was defined as the horizontal and vertical distance of the center of femoral head in relation to the teardrop as described by Russotti and Harris[5]. To differentiate the HHC and anatomical reconstruction, the Ranawat triangle was drawn: (1) a parallel line was drawn above the inter-teardrop line at a distance equal to $20 \%$ of the height of pelvis; (2) a vertical line was drawn $5 \mathrm{~mm}$ lateral to the intersection of the Kohler line and the inter-teardrop line; (3) a diagonal line was drawn superiorly and laterally at an angle of $45^{\circ}$ from the intersection of the vertical line and the inferior horizontal line to intersect the superior horizontal line [10](Fig.3A). The mid-point of the diagonal line was defined as the approximate femoral head center (AFHC)[10]. An acetabular component was considered to be at a high hip center if the center of rotation was higher than AFHC and the anatomical reconstruction was defined as the center of the rotation was lower than AFHC [11]. The four-quadrant system was used to assess cup 
placement, which was proposed by Pagnano et al. [12] and was created by the intersection of a horizontal line and a vertical line through a point $10 \mathrm{~mm}$ superior and lateral to the AFHC (Fig.3B).

Osteolysis was defined as circular or oval areas of distinct bone loss. Radiolucent lines were assessed by DeLee and Charnley classification [13]. Acetabular components were considered loosened if a change of $>3 \mathrm{~mm}$ of migration or $>4^{\circ}$ in the angle of abduction was observed [14].

\section{Clinical evaluation}

All patients were evaluated before the surgery and at the final follow-up using the Harris hip score (HHS), Trendelenburg sign and limp. A standard method was used to check for the Trendelenburg sign [15]. Limp was categorized as slight, moderate and severe [3].

\section{Statistical Analysis}

Statistical analysis was performed using SPSS for Windows Version 25 (IBM, Armonk, NY, USA). Continuous variables were expressed as mean and standard deviation (SD). Categorical data were compared using a $\chi 2$-test. The difference in continuous data between two groups were assessed by Student's t-test. Significance was set at $p<0.05$.

\section{Results}

\section{Morphologic analysis}

The CE angle, Tonnis angle and the width of true acetabular roof are detailed in Table 2. The false acetabulum of Crowe IIIB hips had larger Tonnis angle and smaller CE angle than the Crowe IIIA hips. The width of true acetabular roof was $18.6 \pm 4.3 \mathrm{~mm}$ in the IIIA group and $25.3 \pm 5.0 \mathrm{~mm}$ in the IIIB group $(p<0.001)$. Moreover, the ratio between this width and the height of femoral head demonstrated that the roof of the true acetabulum was significantly thicker in the IIIB hips.

\section{Cup position}

Taking the AFHC as reference, 51 (100\%) IIIA hips and 48 (81.4\%) IIIB hips were reconstructed with high hip center while 11 (18.6\%) IIIB hips were implanted in the anatomical position, of which the center of the rotation was located at $9.0 \pm 2.5 \mathrm{~mm}$ vertically and $22.6 \pm 1.5 \mathrm{~mm}$ horizontally from the bottom of the teardrop.

Of all HHC hips, the mean height of dislocation was $30.4 \pm 3.8 \mathrm{~mm}$ in the IIIA group and $31.8 \pm 3.3 \mathrm{~mm}$ in the IIIB group $(p=0.042)$. The mean vertical distance of center of femoral head in the IIIA group was $33.5 \pm$ $4.5 \mathrm{~mm}$ while it was $31.2 \pm 6.3 \mathrm{~mm}$ in the IIIB group $(p=0.040)$. In addition, the vertical distance of the hip 
center was positively correlated with the height of dislocation in the IIIA group ( $r=0.493, p<0.001)$ (Fig.4). But in the IIIB group, there was no significant correlativity between the two parameters $(r=0.133, p=0.368)$. Moreover, no significant differences were shown regarding the horizontal distance of center of rotation (Table 3).

According to the four-zone system, in the IIIA group, 5 (9.8\%) hips were located in the inferomedial zone, $23(45.1 \%)$ hips in the superomedial zone, $22(43.1 \%)$ hips in the superolateral zone and $1(2.0 \%)$ hip in the inferolateral zone. In the IIIB group, the corresponding numbers were respectively $15(31.3 \%), 17$ (35.4\%), 11 (22.9\%) and 5 (10.4\%) (Fig.5). At the final follow-up, no loosening or progressive radiolucency or osteolysis was observed.

\section{Clinical results}

Of 11 hips which were treated with anatomical reconstruction, the mean HHS was significantly improved from $55.1 \pm 11.0$ to $93.1 \pm 3.3$ at the last follow-up, and no positive Trendelenburg sign or postoperative limp was observed.

Of 51 IIIA hips and 48 IIIB hips which were reconstructed using HHC, a significant improvement in the HHS was also observed. In the IIIA group, the HHS was $52.7 \pm 10.3$ preoperatively and $91.6 \pm 4.3$ at the final follow-up $(p<0.001)$. In the IIIB group, it was improved from $53.2 \pm 12.3$ to $92.9 \pm 3.0(p<0.001)$. Furthermore, 7 IIIA hips and 2 IIIB hips showed a positive Trendelenburg sign ( $p=0.192)$, and 9 patients in the IIIA group and 3 patients in the IIIB group presented with a slight $\operatorname{limp}(p=0.082)$. During the follow-up period, only one IIIB hip underwent the acetabular revision by reason of dislocation at 8.3 years after surgery.

\section{Discussion}

The acetabular reconstruction for Crowe III hips is a complex procedure and brings challenge to orthopedic surgeons because of the deformed acetabulum [16]. We have observed distinct acetabular morphologic variations based on the relationship between the false and the true acetabulum. In addition, we speculated different morphologies may influence the bone mass around the acetabulum, which further affected the cup position. Therefore, in this study, we aimed to 1) assess the acetabular morphologic variations of Crowe III hips by measurement; 2) study the influence of different morphologies on the cup position in THA.

Our measurement on the AP radiographs revealed that the morphology of the false acetabulum was significant different between the two groups. The larger Tonnis angle and the smaller CE angle indicated that the false acetabulum of IIIB hips has larger inclination, which is conducive to accurately classify the deformity. In addition, the superior wall of the true acetabulum in the IIIB group was significant thicker than the IIIA group (Fig. 1). One potential explanation was that the dislocated femoral head of IIIB hips migrated more laterally, resulting in more host bone above the true acetabulum to be preserved. However, 
not all the native bone in the true acetabulum can be effectively used in THA [17]. It is necessary to assess the position of acetabular component in the two group, to determine the influence of the acetabular morphologies.

Without structural bone grafts and any additional augmentation, acetabular reconstruction at the level of the true acetabulum was achieved in $11(18.6 \%)$ hips of the IIIB group but no one in the IIIA group. This result demonstrated more bone mass in the true acetabular roof can provide a better containment for the placement of the cup to facilitate the anatomical reconstruction. Nevertheless, the majority of IIIB hips remained to be reconstructed in an elevated position. From the anatomical perspective, some authors have analyzed the bone stock around the acetabulum and supported the use of HHC technique in Crowe III hips $[18,19,20]$. Liu et al.[21] examined the cup coverage (CC) and rim contact (RC) in patients with Crowe III DDH, and they concluded that CC and RC increased with the peak value at $25 \mathrm{~mm}$ and $22.5 \mathrm{~mm}$ above the native rotation center. Previously, some studies suggested that the $\mathrm{HHC}$ leads to high rates of postoperative complications, including aseptic loosening, dislocation, leg-length discrepancy and limp $[22,23,24,25]$. However, most recent clinical studies demonstrated efficacy of the HHC technique with the use of cementless acetabular cup and ceramic-on-ceramic coupling, which were also utilized in all the hips of this study[26, 27, 28]. In our study, the HHC was used in 51 (100\%) IIIA hips and 48 IIIB (81.4\%) hips. After eliminating the $11 \mathrm{IIIB}$ hips which were reconstructed at the level of the true acetabulum, the height of dislocation was greater in the IIIB group, but the vertical distance of the rotation center was significantly lower than the IIIA group $(31.2 \pm 6.3 \mathrm{~mm}$ vs $33.5 \pm 4.5 \mathrm{~mm}, p=0.040)$, which suggested the acetabulum of IIIB hips can accommodate a well-covered acetabular cup in a relatively lower position. Hence, we believed the extra bone stock in the superior wall of the true acetabulum plays a vital role in lowering the center of rotation.

To eliminate the influence of pelvic size, we performed the further evaluation using the four-zone system which was developed on the basis of AFHC. According to the four-zone system, 15 (31.3\%) IIIB hips were located within the inferomedial quadrant which was the region proximal to the AFHC, while in the IIIA group, there were only $5(9.8 \%)$ hips in this quadrant. When using HHC in the IIIA hips, we had no alternative but to implant the cup in the position of the false acetabulum. But for some IIIB hips, more preserved bone stock located at the junction of true and false acetabulum provided a "middle position" for acetabular component, which can not only permit adequate bone-implant contact, but reduce the height of the center of rotation (Fig. 6). However, the middle position was not always available, and when the bone stock at the junction was insufficient, the cup would only be placed in the position of false acetabulum using HHC. As shown in this study, 11 (22.9\%) IIIB hips were located in the superolateral zone. Nevertheless, compared with 22 (43.1\%) IIIA hips in the superolateral quadrant, it was significantly much less $(p=0.033)$.

The height of dislocation is also an essential factor that influences the placement of acetabular cup. In our study, a significant difference was observed regarding the height of dislocation between the two groups. Through the correlation analysis, the vertical distance of the acetabular cup center was positively correlated with the height of dislocation in the IIIA group. As we described previously, when reconstructing 
the IIIA hip with $\mathrm{HHC}$ technique, the only location for acetabular component was in the false acetabulum. As a result, the higher the height of dislocation, the higher the acetabular component was placed. On the contrary, there was no significant correlation in the IIIB group, because implanting the cup at the junction of the true and the false acetabulum helps lower the cup position.

This study has several limitations. First, the observes found it sometimes difficult to distinguish the relationship between the false and the true acetabulum from the standard pelvic $\mathrm{X}$-ray. Second, we used $\mathrm{HHC}$ technique, which still remained controversial, to reconstruct the majority of hips. However, the HHC have been demonstrated to provide a satisfactory result in many medium- to long-term follow-up studies $[11,26]$. In addition, due to the characteristic of simplicity and effectiveness, many orthopedic surgeons prefer to use the HHC technique. Third, it was a retrospective study, raising the possibility of selection bias. Fourth, our 2D measurement of the morphology of the acetabulum on plain AP radiographs might not reflect the complex three-dimensional geometry around the acetabulum. Based on the CT data, 3D reconstruction and simulated implantation may be a more effective method.

\section{Conclusion}

In summary, through our measurements on the AP radiographs of Crowe III hips, there are distinct morphologic subtypes based on the relationship between the false and the true acetabulum. More bone stock located in the superior wall of the true acetabulum can bring more possibilities for anatomical reconstruction, and lower the height of center of rotation when using the high hip center technique.

\section{Abbreviations}

DDH: Developmental dysplasia of the hip; THA: Total hip arthroplasty; AP: anteroposterior; HHC: High hip center; BMI: body mass index; CE: center-edge; AFHC: approximate femoral head center; HHS: Harris hip score; CC: cup coverage; RC: rim contact.

\section{Declarations}

\section{Ethics approval and consent to participate}

The Ethics Committee of our hospital, General Hospital of Chinese People's Liberation Army, approved the study protocol. A certificate of approval has been provided. The requirement of informed consent was exempted due to the retrospective nature of the study.

\section{Consent for publication}

Not applicable. 


\section{Availability of date and materials}

The data will be made available from the authors upon reasonable request.

\section{Competing interests}

The authors declare that they have no competing interests.

\section{Funding}

No benefits in any form have been received or will be received from a commercial party related directly or indirectly to the subject of this article.

\section{Authors' contributions}

JMS and TZ: Designing the study, Analyzing the data, Writing the manuscript; YGZ and LFG: Designing the study, Editing the manuscript; YD: Collecting the data, Analyzing the data, Reviewing the manuscript; YCZ: Reviewing the literature. All authors have read and approved the final version of this manuscript.

\section{Acknowledgments}

Not applicable.

\section{Author details}

Department of orthopaedics, Chinese People's Liberation Army General Hospital, 28 Fuxing Road, Haidian District, Beijing, China

\section{References}

1. Papachristou G, Hatzigrigoris P, Panousis K, Plessas S, Sourlas J, Levidiotis C, et al. Total hip arthroplasty for developmental hip dysplasia. Int Orthop. 2006;30(1):21-5.

2. Crowe J, Mani V, Ranawat C. Total hip replacement in congenital dislocation and dysplasia of the hip. J Bone Joint Surg Am. 1979;61(1):15-23.

3. Harris W. Traumatic arthritis of the hip after dislocation and acetabular fractures: treatment by mold arthroplasty. An end-result study using a new method of result evaluation. J Bone Joint Surg Am. 1969;51(4):737-55. 
4. Kim M, Kadowaki T. High long-term survival of bulk femoral head autograft for acetabular reconstruction in cementless THA for developmental hip dysplasia. Clin Orthop Relat Res. 2010;468(6):1611-20.

5. Russotti GM, Harris WH. Proximal placement of the acetabular component in total hip arthroplasty. A long-term follow-up study. J Bone Joint Surg Am. 1991;73(4):587-92.

6. Yang Y, Zuo J, Liu T, Xiao J, Liu S, Gao Z. Morphological Analysis of True Acetabulum in Hip Dysplasia (Crowe Classes I-IV) Via 3-D Implantation Simulation. J Bone Joint Surg Am. 2017;99(17):e92.

7. Zheng L-L, Lin Y-Y, Zhang X-Y, Ling Q-H, Liao W-M, Wu P-H. Best bone of acetabulum for cup component placement in Crowe types I to III dysplastic hips. Chinese Medical Journal. 2019;132:2820-6.

8. Bouttier R, Morvan J, Mazieres B, Rat A, Ziegler L, Fardellone P, et al. Reproducibility of radiographic hip measurements in adults. Joint bone spine. 2013;80(1):52-6.

9. Wiberg G. Studies on dysplastic acetabula and congenital subluxation of the hip joint: with special reference to the complication of osteoarthritis. Acta Chir Scand. 1939;83(suppl)58:5-135.

10. Ranawat CS, Dorr LD, Inglis AE. Total hip arthroplasty in protrusio acetabuli of rheumatoid arthritis. $J$ Bone Joint Surg Am. 1980;62(7):1059.

11. Chen M, Luo ZL, Wu KR, Zhang XQ, Ling XD, Shang XF. Cementless Total Hip Arthroplasty With a High Hip Center for Hartofilakidis Type B Developmental Dysplasia of the Hip: Results of Midterm Follow-Up. J Arthroplasty. 2016;31(5):1027-34.

12. Pagnano W, Hanssen AD, Lewallen DG, Shaughnessy WJ. The effect of superior placement of the acetabular component on the rate of loosening after total hip arthroplasty. J Bone Joint Surg Am. 1996;78(7):1004-14.

13. DeLee JG, Charnley J. Radiological demarcation of cemented sockets in total hip replacement. Clin Orthop Relat Res. 1976(121):20-32.

14. Maloney WJ, Galante JO, Anderson M, Goldberg V, Harris WH, Jacobs J, et al. Fixation, polyethylene wear, and pelvic osteolysis in primary total hip replacement. Clin Orthop Relat Res. 1999(369):15764.

15. Hardcastle P, Nade S. The significance of the Trendelenburg test. J Bone Joint Surg Br. 1985;67(5):741-6.

16. Greber EM, Pelt CE, Gililland JM, Anderson MB, Erickson JA, Peters CL. Challenges in Total Hip Arthroplasty in the Setting of Developmental Dysplasia of the Hip. J Arthroplasty. 2017;32(9S):S38S44.

17. van Bosse $\mathrm{H}$, Wedge JH, Babyn P. How are dysplastic hips different? A three-dimensional CT study. Clin Orthop Relat Res. 2015;473(5):1712-23.

18. Komiyama K, Nakashima Y, Hirata M, Hara D, Kohno Y, Iwamoto Y. Does High Hip Center Decrease Range of Motion in Total Hip Arthroplasty? A Computer Simulation Study. J Arthroplasty. 2016;31(10):2342-7. 
19. Antoniades J, Pellegrini V. Cross-sectional anatomy of the ilium: implications for acetabular component placement in total hip arthroplasty. Clin Orthop Relat Res. 2012;470(12):3537-41.

20. Xiao J, Zuo J, Liu P, Qin Y, Li X, Liu T, et al. Cross-sectional Anatomy of Ilium for Guiding Acetabular Component Placement Using High Hip Center Technique in Asian Population. Chinese medical journal. 2015;128(12):1579-83.

21. Liu B, Gao YH, Ding L, Li SQ, Liu JG, Qi X. Computed Tomographic Evaluation of Bone Stock in Patients With Crowe Type III Developmental Dysplasia of the Hip: Implications for Guiding Acetabular Component Placement Using the High Hip Center Technique. J Arthroplasty. 2018;33(3):915-8.

22. Flecher X, Parratte S, Brassart N, Aubaniac JM, Argenson JN. Evaluation of the hip center in total hip arthroplasty for old developmental dysplasia. J Arthroplasty. 2008;23(8):1189-96.

23. Watts CD, Martin JR, Fehring KA, Griffin WL. Inferomedial Hip Center Decreases Failure Rates in Cementless Total Hip Arthroplasty for Crowe II and III Hip Dysplasia. J Arthroplasty. 2018;33(7):2177-81.

24. Karaismailoglu B, Erdogan F, Kaynak G. High Hip Center Reduces the Dynamic Hip Range of Motion and Increases the Hip Load: A Gait Analysis Study in Hip Arthroplasty Patients With Unilateral Developmental Dysplasia. J Arthroplasty. 2019;34(6):1267-72 e1.

25. Watts C, Abdel M, Hanssen A, Pagnano M. Anatomic Hip Center Decreases Aseptic Loosening Rates After Total Hip Arthroplasty with Cement in Patients with Crowe Type-II Dysplasia: A Concise Followup Report at a Mean of Thirty-six Years. J Bone Joint Surg Am. 2016;98(11):910-5.

26. Shen J, Sun J, Ma H, Du Y, Li T, Zhou Y. High Hip Center Technique in Total Hip Arthroplasty for Crowe Type II-III Developmental Dysplasia: Results of Midterm Follow-up. Orthop Surg. 2020;12(4):1245-52.

27. Shen J, Sun J, Du Y, Zhang B, Li T, Zhou Y. Functional and radiographical results of asymmetrically reconstructed total hip arthroplasty in patients with bilateral dysplastic arthritic hips with one hip Crowe II-III and the other Crowe IV: a retrospective cohort study. J Orthop Traumatol. 2021;22(1):11.

28. Galea VP, Laaksonen I, Donahue GS, Fukui K, Kaneuji A, Malchau H, et al. Developmental Dysplasia Treated With Cementless Total Hip Arthroplasty Utilizing High Hip Center Reconstruction: A Minimum 13-Year Follow-up Study. J Arthroplasty. 2018;33(9):2899-905.

\section{Tables}

\section{Table 1}

Characteristic of patients in both groups 


\begin{tabular}{|c|c|c|c|}
\hline Demographic & IIIA & IIIB & $P$ value \\
\hline No. of patients (hips) & $47(51)$ & $54(59)$ & \\
\hline Gender (female/male) & $38 / 9$ & $47 / 7$ & 0.396 \\
\hline Age at THA (years) * & $49.6 \pm 12.1$ & $41.9 \pm 11.1$ & 0.001 \\
\hline Height $(\mathrm{cm})^{*}$ & $160.8 \pm 8.6$ & $159.8 \pm 6.6$ & 0.520 \\
\hline Body weight $(\mathrm{kg})^{*}$ & $62.7 \pm 10.8$ & $59.7 \pm 8.6$ & 0.113 \\
\hline BMI $\left(\mathrm{kg} / \mathrm{m}^{2}\right)^{*}$ & $24.2 \pm 3.5$ & $23.4 \pm 3.3$ & 0.207 \\
\hline Follow-up period (years) * & $5.1 \pm 2.8$ & $6.2 \pm 2.9$ & 0.065 \\
\hline
\end{tabular}

Table 2

Measurement of acetabular morphological variations

\begin{tabular}{|llll|}
\hline Parameter & IIIA $(\mathrm{n}=51)$ & IIIB $(\mathrm{n}=59)$ & P value \\
\hline CE angle $\left(^{\circ}\right)$ & $9.3 \pm 21.3$ & $-1.1 \pm 19.4$ & 0.009 \\
\hline Tonnis angle $\left(^{\circ}\right)$ & $30.3 \pm 6.6$ & $49.0 \pm 9.8$ & $<0.001$ \\
\hline Width of the roof of T-Ace $(\mathrm{mm})$ & $18.6 \pm 4.3$ & $25.3 \pm 5.0$ & $<0.001$ \\
\hline The ratio of width of the roof & $0.48 \pm 0.11$ & $0.66 \pm 0.12$ & $<0.001$ \\
\hline CE, center-edge; T-Ace, true acetabulum. & & \\
\hline
\end{tabular}

\section{Table 3}

Radiographic evaluation of all $\mathrm{HHC}$ hips 


\begin{tabular}{lccc} 
Parameter & IIIA $(\mathrm{n}=51)$ & IIIB $(\mathrm{n}=48)$ & P value \\
\hline Vertical distance * & $33.5 \pm 4.5$ & $31.2 \pm 6.3$ & 0.040 \\
\hline Horizontal distance * & $31.8 \pm 4.6$ & $31.9 \pm 6.2$ & 0.896 \\
\hline Height of dislocation * & $30.4 \pm 3.8$ & $31.8 \pm 3.3$ & 0.042 \\
\hline Postoperative zone & & & \\
\hline Zone 1 & $5(9.8 \%)$ & $15(31.3 \%)$ & 0.008 \\
\hline Zone 2 & $23(45.1 \%)$ & $17(35.4 \%)$ & 0.327 \\
Zone 3 & $22(43.1 \%)$ & $11(22.9 \%)$ & 0.033 \\
\hline Zone 4 & $1(2.0 \%)$ & $5(10.4 \%)$ & 0.089 \\
\hline * Values given as mean \pm standard deviation. &
\end{tabular}

Figures 


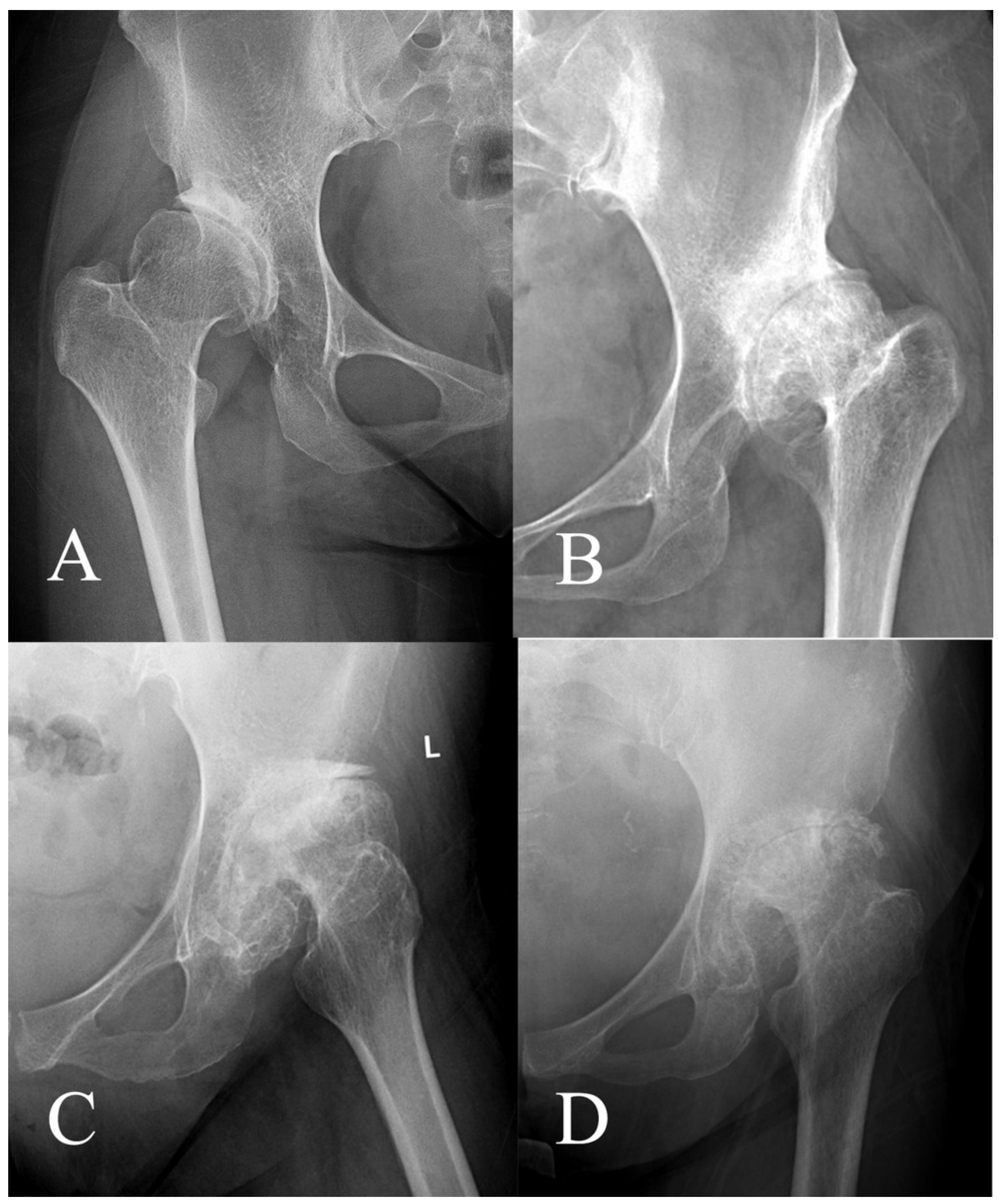

\section{Figure 1}

The morphologic variations of the deformed acetabulum in the Crowe III hips. A-B: The crest between the false and the true acetabulum can be significantly observed. C-D: The junction between the false and the true acetabulum was indistinct. 


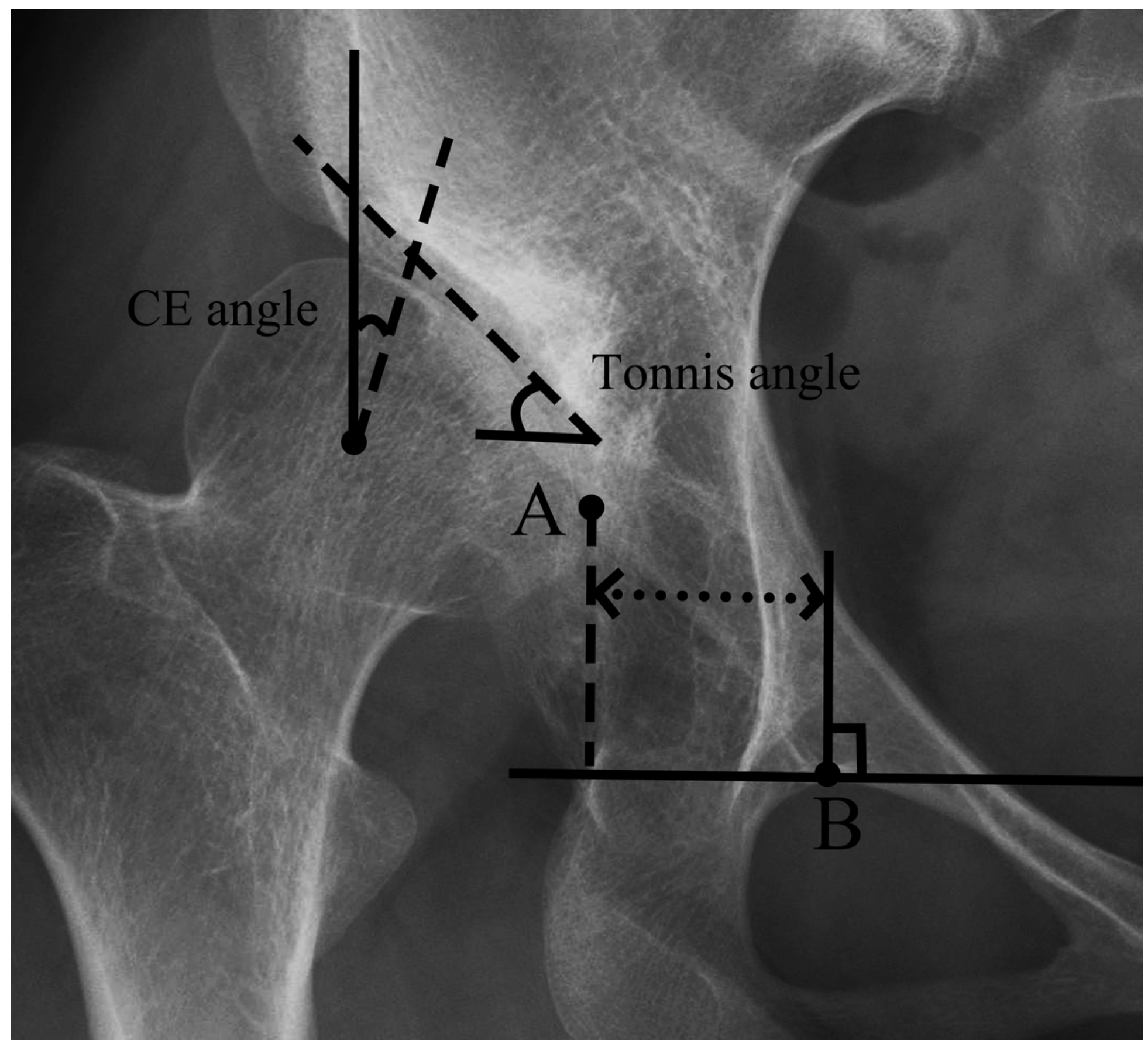

\section{Figure 2}

The measurement of the $\mathrm{CE}$ angle, Tonnis angle and the width of true acetabular roof. Point A refers to the most superior point of the true acetabulum. Point B refers to the most inferior point of the teardrop. 


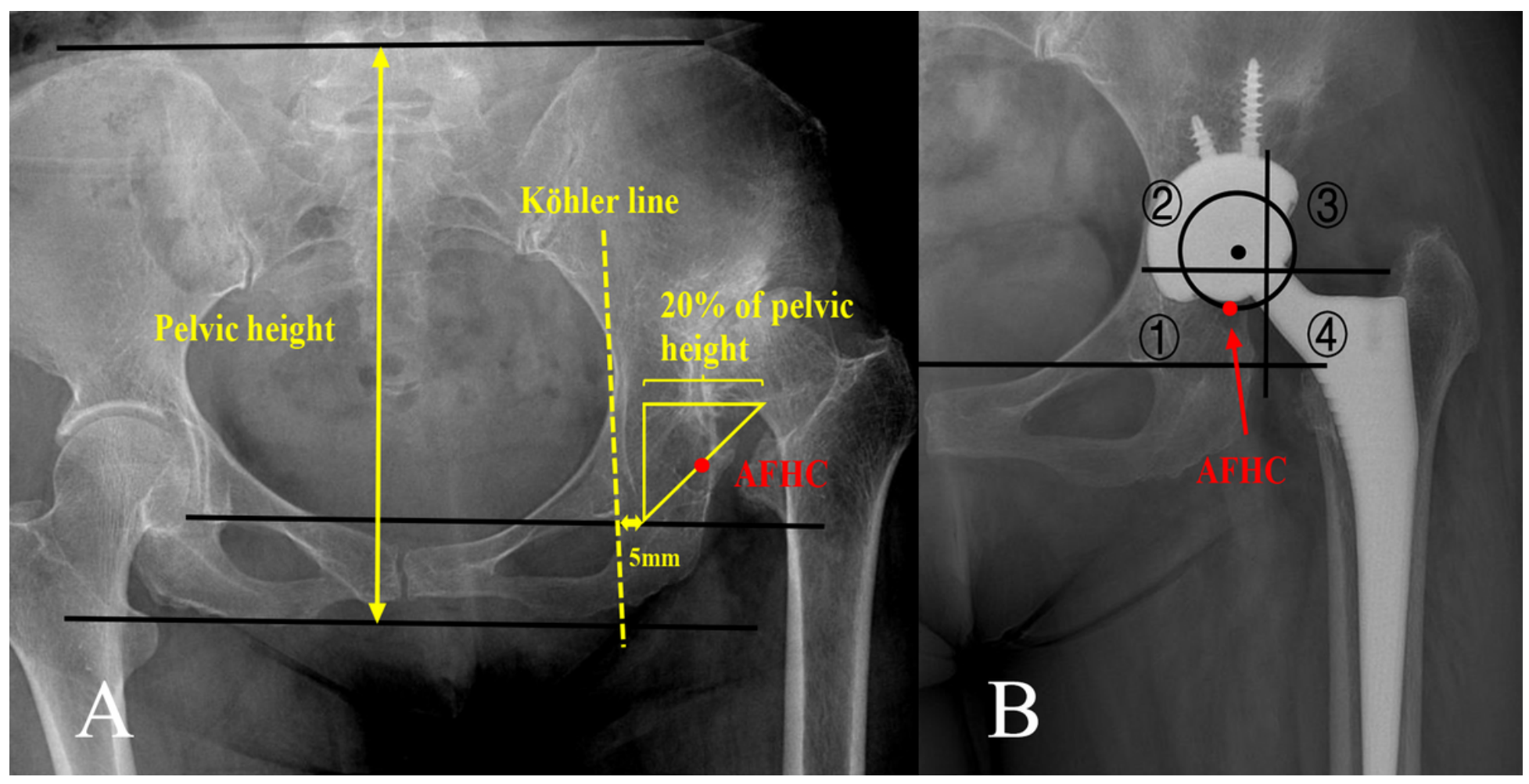

\section{Figure 3}

(A) The AFHC is located along the hypotenuse of an equilateral right triangle, whose inferomedial corner is located $5 \mathrm{~mm}$ lateral to the point of intersection between the inter-teardrop line and Kohler line, and whose vertical and horizontal distance are equal to $20 \%$ of pelvic height. (B) The four-zone system is created by the intersection of a horizontal line and a vertical line through a point $10 \mathrm{~mm}$ superior and lateral to the AFHC. 


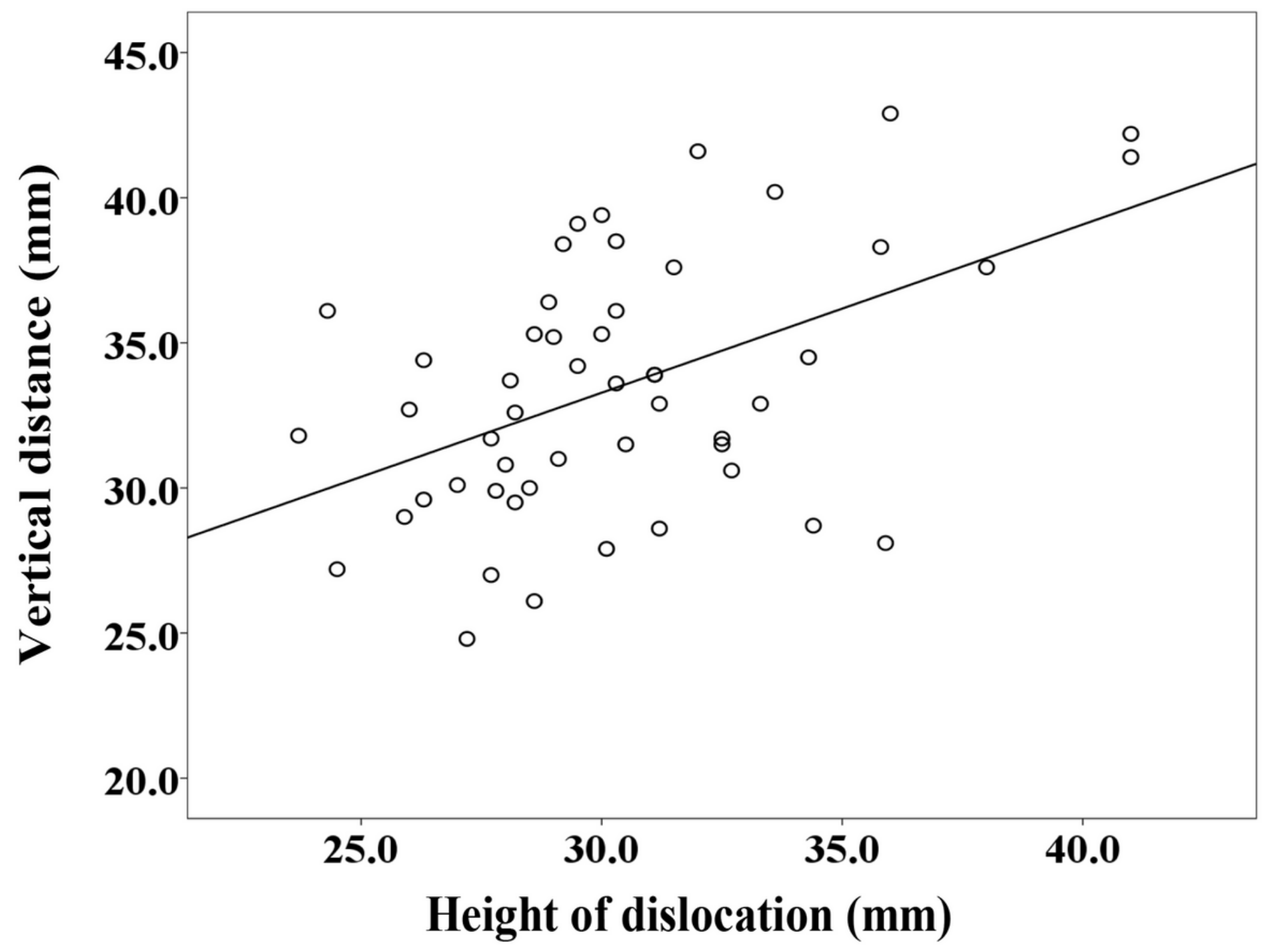

Figure 4

Correlation between the vertical distance of center of femoral head and the height of dislocation in the IIIA group $(r=0.493, p<0.001)$. 


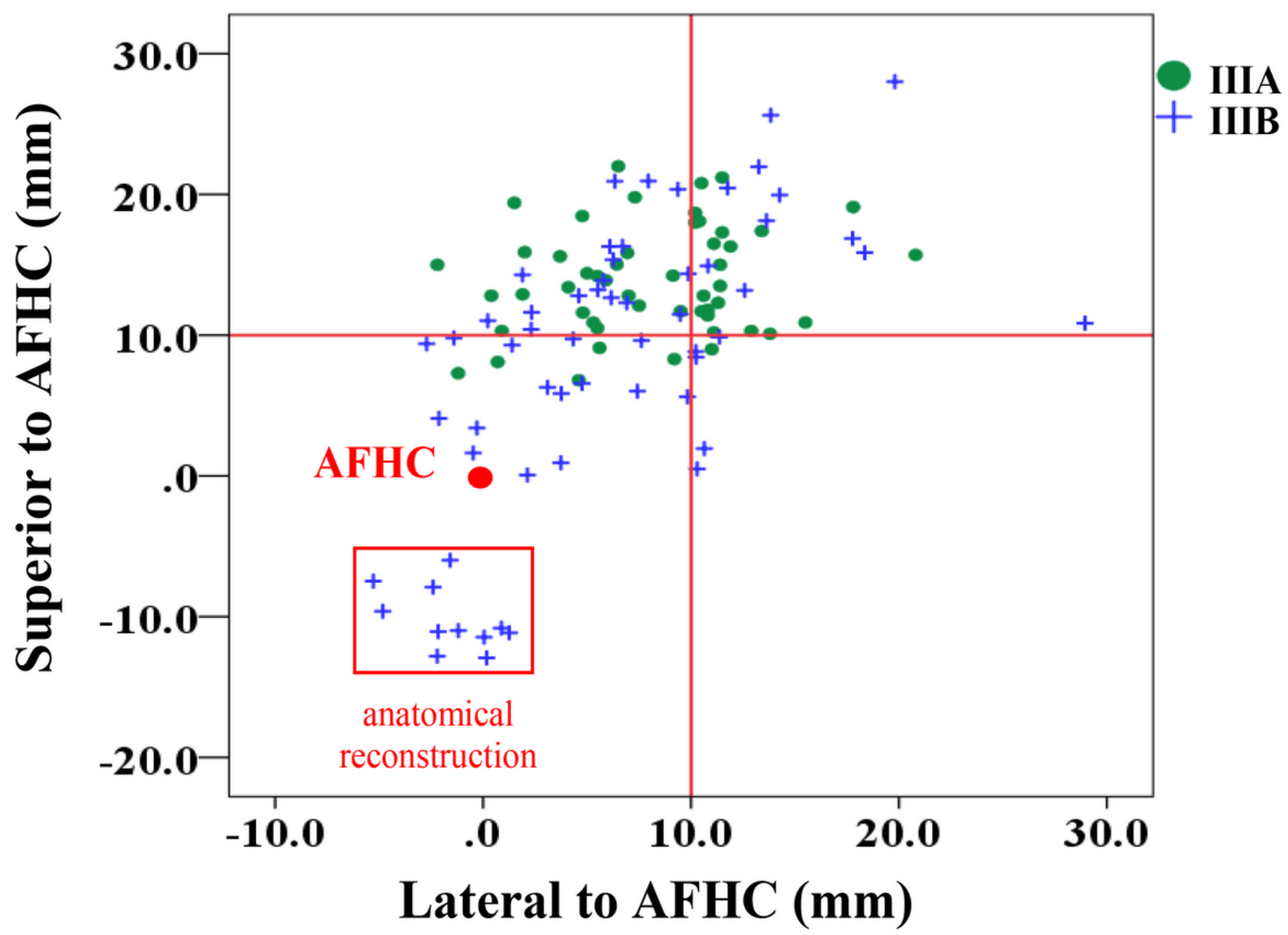

Figure 5

Scatterplot of the distribution of hip centers relative to the AFHC. The red solid line represents the fourzone system. 


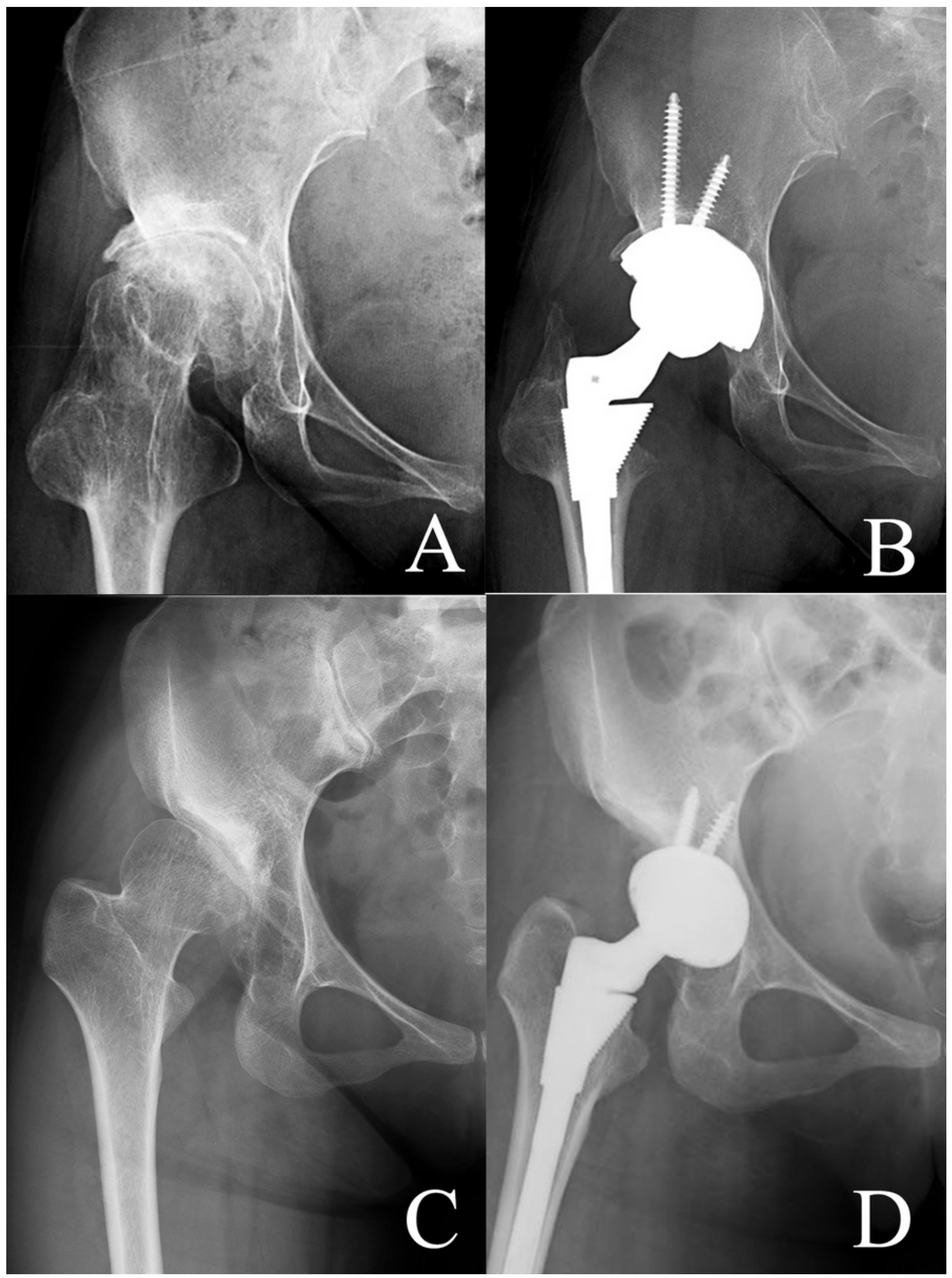

\section{Figure 6}

Preoperative AP radiograph (A) showed a Crowe IIIA hip with extensive bone deficiency in the superior wall of the true acetabulum, and the postoperative AP film (B) showed the acetabular cup was implanted in the position of the false acetabulum using high hip center technique. Preoperative AP radiograph (C) showed a Crowe IIIB hip with more bone stock located in the roof of the true acetabulum. The acetabular cup was placed at the junction of the false and true acetabulum (D). 